\title{
Dynamic risk measure for BSVIE with jumps and semimartingale issues
}

\author{
Nacira Agram ${ }^{1,2}$
}

22 December 2018

\begin{abstract}
Risk measure is a fundamental concept in finance and in the insurance industry, it is used to adjust life insurance rates. In this current paper, we will study dynamic risk measures by means of backward stochastic Volterra integral equations (BSVIEs) with jumps. We prove a comparison theorem for such a type of equations. Since the solution of a BSVIEs is not a semimartingale in general, we will discuss some particular semimartingale issues.
\end{abstract}

$\operatorname{MSC}(2010)$ :

$60 \mathrm{H} 07,60 \mathrm{H} 20,60 \mathrm{H} 30,45 \mathrm{D} 05,45 \mathrm{R} 05$.

Keywords: Brownian motion, compensated Poisson random measure, backward stochastic Volterra integral equation, risk measure, semimartingale.

\section{Introduction}

Consider the solution couple $(Y, Z)$ of the following nonlinear backward stochastic differential equation (BSDE)

$$
d Y(t)=-F(t, Y(t), Z(t)) d t+Z(t) d B(t), Y(T)=-\xi .
$$

\footnotetext{
${ }^{1}$ Department of Mathematics, University of Oslo, P.O. Box 1053 Blindern, N-0316 Oslo, Norway and University of Biskra, Algeria. Email: naciraa@math.uio.no.

${ }^{2}$ This research was carried out with support of the Norwegian Research Council, within the research project Challenges in Stochastic Control, Information and Applications (STOCONINF), project number 250768/F20.

Part of this work has been done while the author is visiting the University of Alberta, Canada and I would like to thank Prof. Hu for the hospitality and his comments during my talk.
} 
It is known that under mild conditions this equation has a unique solution. This was first proved by Pardoux and Peng [13], for a Lipschitz driver $F$ and a square integrable random variable $\xi$. Such a type of equation first appeared in its linear form as an adjoint equation when Bismut [5] studied the stochastic version of the Pontryagin's maximum principle. Due to their significant applications to finance and insurance, BSDEs have gained a lot of interest since 1990. See e.g. the seminal work by El Karoui et al [7] for more details. Subsequently, it was discovered that BSDEs could also be used to represent risk measures. We recall this connection briefly. First, let us recall the definition of a convex risk measure, see for instance Föllmer and Schied [8], Frittelli and Rosazza Gianin [9].

Definition 1.1 A convex risk measure is a map $\rho: L^{p}\left(\mathcal{F}_{T}\right) \rightarrow \mathbb{R}, p \in[2, \infty]$ that satisfies the following properties:

- (Convexity) $\rho\left(\lambda \varphi_{1}+(1-\lambda) \varphi_{2}\right) \leq \lambda \rho\left(\varphi_{1}\right)+(1-\lambda) \rho\left(\varphi_{2}\right)$ for all $\lambda \in[0,1]$ and all $\varphi_{1}, \varphi_{2} \in L^{p}\left(\mathcal{F}_{T}\right)$.

- (Monotonicity) If $\varphi_{1} \leq \varphi_{2}$, then $\rho\left(\varphi_{1}\right) \geq \rho\left(\varphi_{2}\right)$.

- (Translation invariance) $\rho(\varphi+a)=\rho(\varphi)-a$ for all $\varphi \in L^{p}\left(\mathcal{F}_{T}\right)$ and all constants $a$.

- $\rho(0)=0$.

The last axiom is known as a normalisation and usually it is assumed for convenience. The construction of risk measures from solutions to BSDEs is given as follows:

Let $Y(t)=Y^{-\xi}(t)$ be the first component of the solution of the BSDE (1.1). Assume that the driver $F(t, y, z)$ of the BSDE (1.1) does not depend on $y$ and that $z \mapsto F(t, z)$ is convex for all $t$. Then

$$
\rho(\xi):=Y^{-\xi}(0)
$$

defines a convex risk measure. This shows how crucial is the choice of the functional $F$. This relation extends to the concept of dynamic risk measure. See for example Barrieu and El Karoui [4], Quenez and Sulem [16]. Peng [14], Rosazza Gianin [18] have used the notion of the nonlinear expectation $(g$-BSDE) to represent a dynamic risk measures.

The connection above has also been generalised to BSDEs with jumps by Quenez and Sulem [15] and Øksendal and Sulem [12]. Yong [26] extended the notion of dynamic risk measures by means of BSDEs to a specific class of dynamic risk measures that arise as a solutions to the so-called BSVIEs, as follows: Consider a position (wealth) process $\psi \in L_{\mathcal{F}_{T}}^{2}(0, T)$ instead of a random variable $\xi$ and let the couple $(Y(t), Z(t, s))$ be the solution of the following BSVIE

$$
Y(t)=-\psi(t)+\int_{t}^{T} g(t, s, Y(s), Z(t, s)) d s-\int_{t}^{T} Z(t, s) d B(s), t \in[0, T] .
$$

More generally, he considers the BSVIE

$$
Y(t)=-\psi(t)+\int_{t}^{T} g(t, s, Y(s), Z(t, s), Z(s, t)) d s-\int_{t}^{T} Z(t, s) d B(s), t \in[0, T] .
$$


We may remark here, the difference between the two above equations is that the driver in (1.3) depends on both $Z(t, s)$ and $Z(s, t)$. This type of equations appeared in its linear form when Yong [23] proved the duality principle between linear forward stochastic Volterra integral equations (SVIEs) and linear BSVIEs.

Moreover, differentiability of BSDEs and BSVIEs were used for the purpose of capital allocation for risk measures by Kromer and Overbeck [11]. We recall now the definition of the dynamic risk measure by means of BSVIEs from Yong [26].

Definition 1.2 A map $\rho:[0, T] \times L_{\mathcal{F}_{T}}^{2}(0, T) \rightarrow L_{\mathbb{F}}^{2}(0, T)$ is called a dynamic risk measure if the following hold:

- (Past independence) For any $\psi(\cdot), \psi^{\prime}(\cdot) \in L_{\mathcal{F}_{T}}^{2}(0, T)$, if

$$
\psi(s)=\psi^{\prime}(s), \quad \text { a.s. }, \omega \in \Omega, s \in[t, T],
$$

for some $t \in[0, T)$, then

$$
\rho(t ; \psi(\cdot))=\rho\left(t ; \psi^{\prime}(\cdot)\right), \quad \text { a.s. }, \omega \in \Omega .
$$

- (Monotonicity) For any $\psi(\cdot), \psi^{\prime}(\cdot) \in L_{\mathcal{F}_{T}}^{2}(0, T)$, if

$$
\psi(s) \leq \psi^{\prime}(s), \quad \text { a.s. }, \omega \in \Omega, s \in[t, T]
$$

for some $t \in[0, T)$, then

$$
\rho(t ; \psi(\cdot)) \geq \rho\left(t ; \psi^{\prime}(\cdot)\right), \quad \text { a.s. }, \omega \in \Omega .
$$

Definition 1.3 A dynamic risk measure $\rho: L_{\mathcal{F}_{T}}^{2}(0, T) \rightarrow L_{\mathbb{F}}^{2}(0, T)$ is called a convex risk measure if

- (Convexity) For any $\psi(\cdot), \psi^{\prime}(\cdot) \in L_{\mathcal{F}_{T}}^{2}(0, T)$ and $\lambda \in[0,1]$,

$$
\rho\left(t ; \lambda \psi(\cdot)+(1-\lambda) \psi^{\prime}(\cdot)\right) \leq \lambda \rho(t ; \psi(\cdot))+(1-\lambda) \rho\left(t ; \psi^{\prime}(\cdot)\right), \quad \text { a.s., } \omega \in \Omega, t \in[0, T] .
$$

- (Translation invariance) For any $\psi(\cdot)$ and any constant $a$ it holds that

$$
\rho(t ; \psi(\cdot)+a)=\rho(t ; \psi(\cdot))-a, \quad \text { a.s. }, \omega \in \Omega, s \in[t, T],
$$

This type of equation can also be used as a model for recursive utility, because the equivalent formulation of (1.2) is

$$
Y(t)=\mathbb{E}\left[-\psi(t)+\int_{t}^{T} g(t, s, Y(s), Z(t, s)) d s \mid \mathcal{F}_{t}\right] .
$$

This can be regarded as an extension of the classical recursive utility concept of Duffie and Epstein [6] to systems with memory. For example, for the consumption process $c(t) \geq 0$, we consider its recursive utility process $Y(t)$ defined by

$$
Y(t)=\mathbb{E}\left[-\psi(t)+\int_{t}^{T} g(t, s, Y(s), Z(t, s), c(s)) d s \mid \mathcal{F}_{t}\right] .
$$


For more details about BSVIEs and their applications, we refer to Yong et al [23], [24], [25] and to Agram et al [2],[3], [1]. In Wang and Yong [21] comparison theorems for different classes of BSVIEs have been proved. In all the above works, only BSVIEs driven by Brownian motion are considered.

In the current paper we are interested in BSVIEs with jumps of the form

$$
\begin{gathered}
Y(t)=-\psi(t)+\int_{t}^{T} g(t, s, Y(s), Z(t, s), K(t, s, \cdot)) d s-\int_{t}^{T} Z(t, s) d B(s) \\
-\int_{t}^{T} \int_{\mathbb{R}_{0}} K(t, s, \zeta) \tilde{N}(d s, d \zeta), t \in[0, T]
\end{gathered}
$$

where $B$ is a standard Brownian motion and $\tilde{N}$ is an independent compensated Poisson random measure (see below for definitions). For a Lipschitz driver, Agram et al [2] have proved that there exists a unique solution of equation (1.4). Under weaker assumptions (non-Lipschitz driver), we refer to Wang and Zhang [22] and to Ren [17].

The general nature of the BSVIEs does not allow us to write explicitly the solution of a linear BSVIEs in general. However, $\mathrm{Hu}$ and Øksendal [10] have obtained a closed solution formula for a special class of linear BSVIEs with jumps. Using their result, we will prove comparison theorems for BSVIEs with jumps.

The main contributions in this paper is the extension to jumps of the comparison theorems in Wang and Yong [21] and also the dynamic measures by BSVIEs with jumps of the paper by Yong [26].

Moreover, due to the dependence on $t$ in the coefficients, such BSVIEs are complicated to deal with. It is not even clear if the solution of a BSVIE is a semimartingale in general. We will discuss some particular cases where the solution can be a semimartingale.

Here is an outline of our paper:

In Section 2 we give some preliminaries on BSVIEs with jumps. Then in Section 3 we prove a comparison theorem for BSVIEs with jumps. In Section 4 we study dynamic risk measures by means of BSVIEs with jumps. Finally, in Section 5 we discuss some semimartingale issues for BSVIEs.

\section{Preliminaries on BSVIEs with jumps}

Let $(\Omega, \mathcal{F}, \mathbb{P})$ be a given probability space with filtration $\mathbb{F}=\left(\mathcal{F}_{t}\right)_{t \geq 0}$ generated by a onedimensional Brownian motion $B$ and an independent Poisson random measure $N(d t, d \zeta)$. Let $\nu(d \zeta) d t$ denote the Lévy measure of $N$, and let $\tilde{N}(d t, d \zeta)$ denote the compensated Poisson random measure $N(d t, d \zeta)-\nu(d \zeta) d t$.

Put $\triangle:=\left\{(t, s) \in[0, T]^{2}: t \leq s\right\}$. We define the following sets:

- $L_{y}^{2}$ consists of the $\mathbb{F}$-adapted càdlàg processes $Y:[0, T] \times \Omega \rightarrow \mathbb{R}$ equipped with the norm

$$
\|Y\|_{L_{y}^{2}}^{2}:=\mathbb{E}\left[\int_{0}^{T}|Y(t)|^{2} d t\right]<\infty
$$


- $L_{z}^{2}$ consists of the $\mathbb{F}$-predictable processes

$$
Z: \triangle \times \Omega \rightarrow \mathbb{R}
$$

such that $\mathbb{E}\left[\int_{0}^{T} \int_{t}^{T}|Z(t, s)|^{2} d s d t\right]<\infty$ with $s \mapsto Z(t, s)$ being $\mathbb{F}$-predictable on $[t, T]$. We equip $L_{z}^{2}$ with the norm

$$
\|Z\|_{L_{z}^{2}}^{2}:=\mathbb{E}\left[\int_{0}^{T} \int_{t}^{T}|Z(t, s)|^{2} d s d t\right]
$$

- $L_{\nu}^{2}$ consists of all Borel functions $K: \mathbb{R}_{0} \rightarrow \mathbb{R}$, such that

$$
\|K\|_{L_{\nu}^{2}}^{2}:=\int_{\mathbb{R}_{0}} K(t, s, \zeta)^{2} \nu(d \zeta)<\infty
$$

- $H_{\nu}^{2}$ consists of $\mathbb{F}$-predictable processes $K: \triangle \times \mathbb{R}_{0} \times \Omega \rightarrow \mathbb{R}$, such that

$$
\mathbb{E}\left[\int_{0}^{T} \int_{t}^{T} \int_{\mathbb{R}_{0}}|K(t, s, \zeta)|^{2} \nu(d \zeta) d s d t\right]<\infty
$$

and $s \mapsto K(t, s, \cdot)$ being $\mathbb{F}$-predictable on $[t, T]$. We equip $H_{\nu}^{2}$ with the norm

$$
\|K\|_{H_{\nu}^{2}}^{2}:=\mathbb{E}\left[\int_{0}^{T} \int_{t}^{T} \int_{\mathbb{R}_{0}}|K(t, s, \zeta)|^{2} \nu(d \zeta) d s d t\right] .
$$

- Let $L_{\mathcal{F}_{T}}^{2}[0, T]$ be the space of all processes $\psi:[0, T] \times \Omega \rightarrow \mathbb{R}$ and $\psi$ is $\mathcal{F}_{T^{-}}$-measurable for all $t \in[0, T]$, such that

$$
\|\psi\|_{L_{\mathcal{F}_{T}}^{2}[0, T]}^{2}=\mathbb{E}\left[\int_{0}^{T}|\psi(t)|^{2} d t\right]<\infty
$$

- $L_{\mathbb{F}}^{2}[0, T]$ is the space of all $\psi \in L_{\mathcal{F}_{T}}^{2}[0, T]$ that are $\mathbb{F}$-adapted.

We are interested in the $\operatorname{BSVIE}(Y, Z, K) \in L_{y}^{2} \times L_{z}^{2} \times H_{\nu}^{2}$, given by

$$
\begin{gathered}
Y(t)=\psi(t)+\int_{t}^{T} g(t, s, Y(s), Z(t, s), K(t, s, \cdot)) d s-\int_{t}^{T} Z(t, s) d B(s) \\
-\int_{t}^{T} \int_{\mathbb{R}_{0}} K(t, s, \zeta) \tilde{N}(d s, d \zeta), t \in[0, T]
\end{gathered}
$$

where the following conditions are satisfied:

(i) The driver $g: \triangle \times \mathbb{R}^{2} \times L_{\nu}^{2} \times \Omega \rightarrow \mathbb{R}$ satisfies the following integrability condition:

$$
\mathbb{E}\left[\int_{0}^{T}\left(\int_{t}^{T} g(t, s, 0,0,0) d s\right)^{2} d t\right]<+\infty
$$


(ii) The driver $g$ satisfies the following Lipschitz condition:

There exists a constant $C>0$, such that, for all $(t, s) \in \triangle$,

$$
\begin{aligned}
& \left|g(t, s, y, z, k(\cdot))-g\left(t, s, y^{\prime}, z^{\prime}, k^{\prime}(\cdot)\right)\right| \\
& \leq C\left(\left|y-y^{\prime}\right|+\left|z-z^{\prime}\right|+\left(\int_{\mathbb{R}_{0}}\left|k(\zeta)-k^{\prime}(\zeta)\right|^{2} \nu(d \zeta)\right)^{\frac{1}{2}}\right)
\end{aligned}
$$

for all $y, y^{\prime}, z, z^{\prime} \in \mathbb{R}, k(\cdot), k^{\prime}(\cdot) \in L_{\nu}^{2}$.

(iii) The terminal condition $\psi(\cdot) \in L_{\mathcal{F}_{T}}^{2}[0, T]$.

For this equation the following is known:

Theorem 2.1 (Agram et al [2]) Under the above assumptions, there exists a unique solution $(Y, Z, K) \in L_{y}^{2} \times L_{z}^{2} \times H_{\nu}^{2}$ of the BSVIEs with jumps (2.1), with

$$
\|(Y, Z, K)\|_{L_{y}^{2} \times L_{z}^{2} \times H_{\nu}^{2}}^{2} \leq C \mathbb{E}\left[|\psi(t)|^{2}+\left(\int_{t}^{T} g(t, s, 0,0,0) d s\right)^{2}\right] .
$$

In the next section we will prove a comparison theorem for some BSVIEs by using the linearisation of the solutions.

\section{Comparison theorem for BSVIEs with jumps}

In order to be able to prove the comparison theorem for BSVIEs with jumps, let us first recall the closed formula for linear BSVIEs with jumps. Consider the solution triplet $(Y, Z, K) \in$ $L_{y}^{2} \times L_{z}^{2} \times H_{\nu}^{2}$ of the following linear BSVIE

$$
\begin{aligned}
Y(t):=\psi & (t)+\int_{t}^{T}\left[\alpha(t, s) Y(s)+\beta(s) Z(t, s)+\int_{\mathbb{R}_{0}} \theta(s, \zeta) K(t, s, \zeta) \nu(d \zeta)\right] d s \\
& -\int_{t}^{T} Z(t, s) d B(s)-\int_{t}^{T} \int_{\mathbb{R}_{0}} K(t, s, \zeta) \tilde{N}(d s, d \zeta), t \in[0, T]
\end{aligned}
$$

where $\psi(\cdot) \in L_{\mathcal{F}_{T}}^{2}[0, T], \alpha(t, s) ; 0 \leq t \leq s \leq T$ and $\left(\beta(s), \theta(s, \zeta) ; 0 \leq s \leq T, \zeta \in \mathbb{R}_{0}\right.$ are given (deterministic) measurable functions of $t, s$ and $\zeta$, with values in $\mathbb{R}$. For simplicity we assume that these functions are bounded, and we assume that there exists $\varepsilon>0$ such that $\theta(s, \zeta) \geq-1+\varepsilon$ for all $s, \zeta$.

Define the measure $\mathbb{Q}$ by

$$
d \mathbb{Q}=M(T) d \mathbb{P} \text { on } \mathcal{F}_{T},
$$

where

$$
\begin{aligned}
M(t) & :=\exp \left(\int_{0}^{t} \beta(s) d B(s)-\frac{1}{2} \int_{0}^{t} \beta^{2}(s) d s\right. \\
& +\int_{0}^{t} \int_{\mathbb{R}_{0}} \ln (1+\theta(s, \zeta)) \tilde{N}(d s, d \zeta) \\
& \left.+\int_{0}^{t} \int_{\mathbb{R}_{0}}\{\ln (1+\theta(s, \zeta))-\theta(s, \zeta)\} \nu(d \zeta) d s\right) .
\end{aligned}
$$

Then under the new measure $\mathbb{Q}$ the process

$$
B_{\mathbb{Q}}(t):=B(t)-\int_{0}^{t} \beta(s) d s, \quad 0 \leq t \leq T .
$$


is a Brownian motion, and the random measure

$$
\tilde{N}_{\mathbb{Q}}(d t, d \zeta):=\tilde{N}(d t, d \zeta)-\theta(t, \zeta) \nu(d \zeta) d t
$$

is the $\mathbb{Q}$-compensated Poisson random measure of $N(\cdot, \cdot)$, in the sense that the process

$$
\tilde{N}_{\gamma}(t):=\int_{0}^{t} \int_{\mathbb{R}_{0}} \gamma(s, \zeta) \tilde{N}_{\mathbb{Q}}(d s, d \zeta)
$$

is a local $\mathbb{Q}$-martingale, for all predictable processes $\gamma(t, \zeta)$ such that

$$
\int_{0}^{T} \int_{\mathbb{R}_{0}} \gamma^{2}(t, \zeta) \theta^{2}(t, \zeta) \nu(d \zeta) d t<\infty
$$

By this change of measure the equation (3.1) is equivalent to

$$
Y(t)=\psi(t)+\int_{t}^{T} \alpha(t, s) Y(s) d s-\int_{t}^{T} Z(t, s) d B_{\mathbb{Q}}(s)-\int_{t}^{T} \int_{\mathbb{R}_{0}} K(t, s, \zeta) \tilde{N}_{\mathbb{Q}}(d s, d \zeta) .
$$

For all $0 \leq t \leq r \leq T$, define

$$
\alpha^{(1)}(t, r)=\alpha(t, r), \quad \alpha^{(2)}(t, r)=\int_{t}^{r} \alpha(t, s) \alpha(s, r) d s
$$

and inductively

$$
\alpha^{(n)}(t, r)=\int_{t}^{r} \alpha^{(n-1)}(t, s) \alpha(s, r) d s, n=3,4, \cdots .
$$

Note that if $|\alpha(t, r)| \leq C$ (constant) for all $t, r$, then by induction on $n \in \mathbb{N}$

$$
\left|\alpha^{(n)}(t, r)\right| \leq \frac{C^{n} T^{n}}{n !}
$$

for all $t, r, n$. Put,

$$
\Psi(t, r):=\Sigma_{n=1}^{\infty}\left|\alpha^{(n)}(t, r)\right|<\infty,
$$

for all $t, r$. Then we have the following:

Theorem 3.1 ( $\mathrm{Hu}$ and $\varnothing \mathrm{ksendal}[\mathbf{1 0}])$ The first component $Y(t)$ of the solution of the linear BSVIE with jumps (3.1) is given by

$$
Y(t)=\frac{\mathbb{E}\left[\frac{d \mathbb{Q}}{d \mathbb{P}}\left\{\psi(t)+\int_{t}^{T} \Phi(t, r) \psi(r) d r\right\} \mid \mathcal{F}_{t}\right]}{\mathbb{E}\left[\frac{d \mathbb{Q}}{d \mathbb{P}} \mid \mathcal{F}_{t}\right]} .
$$

Remark 3.2 In the previous theorem, we can get the same result by considering predictable processes $\left(\beta(s), \theta(s, \zeta) ; 0 \leq s \leq T, \zeta \in \mathbb{R}_{0}\right)$ instead of deterministic functions since the Girsanov change of measure theorem is still valid.

We now state and prove the comparison theorem. 
Theorem 3.3 (Comparison Theorem) For $i=1,2$, let $g_{i}: \triangle \times \mathbb{R}^{2} \times L_{\nu}^{2} \times \Omega \rightarrow \mathbb{R}$ and $\psi_{1}(t), \psi_{2}(t) \in L_{\mathcal{F}_{T}}^{2}[0, T]$ and let $\left(Y^{i}, Z^{i}, K^{i}\right)_{i=1,2}$ be the solutions of

$$
\begin{gathered}
Y^{i}(t)=\psi^{i}(t)+\int_{t}^{T} g_{i}\left(t, s, Y^{i}(s), Z^{i}(t, s), K^{i}(t, s, \cdot)\right) d s-\int_{t}^{T} Z^{i}(t, s) d B(s) \\
-\int_{t}^{T} \int_{\mathbb{R}_{0}} K^{i}(t, s, \zeta) \tilde{N}(d s, d \zeta), t \in[0, T] .
\end{gathered}
$$

Assume that the drivers $\left(g_{i}\right)_{i=1,2}$ are Lipschitz and satisfy

$$
g_{1}\left(t, s, y^{2}, z^{2}, k^{2}\right) \geq g_{2}\left(t, s, y^{2}, z^{2}, k^{2}\right), \forall t, \mathbb{P} \text {-a.s. },
$$

and that there exists a bounded predictable process $\theta(s, t, \zeta)$ and $a \Pi(\cdot) \in L_{\nu}^{2}$ such that $d s \otimes d \mathbb{P} \otimes \nu(d \zeta)$-a.s.

$$
\theta(s, \zeta) \geq-1+\varepsilon \text { and }|\theta(s, \zeta)| \leq \Pi(\zeta),
$$

and the following inequality holds

$$
\begin{gathered}
g_{1}\left(t, s, Y^{2}(s), Z^{2}(t, s), K^{1}(t, s, \cdot)\right)-g_{1}\left(t, s, Y^{2}(s), Z^{2}(t, s), K^{2}(t, s, \cdot)\right) \\
\geq \int_{\mathbb{R}_{0}} \theta(s, \zeta)\left(K^{1}(t, s, \zeta)-K^{2}(t, s, \zeta)\right) \nu(d \zeta)
\end{gathered}
$$

Moreover, assume that the driver $g$ is increasing on $y$, such that

$$
g\left(t, s, y^{1}, z, k\right) \geq g\left(t, s, y^{2}, z, k\right), \text { if } y^{1} \geq y^{2}, \forall t, \mathbb{P} \text {-a.s. }
$$

and

$$
\psi_{1}(t) \geq \psi_{2}(t) \text { for each } t \in[0, T], \mathbb{P} \text {-a.s. }
$$

Then $Y^{1}(t) \geq Y^{2}(t) \mathbb{P}$-a.s. for each $t$.

Proof We set

$$
\begin{aligned}
\hat{\psi} & =\psi_{1}-\psi_{2}, \\
\hat{Y} & =Y^{1}-Y^{2}, \hat{Z}=Z^{1}-Z^{2}, \hat{K}=K^{1}-K^{2},
\end{aligned}
$$

we have

$$
\begin{gathered}
\hat{Y}(t)=\hat{\psi}(t)+\int_{t}^{T}\left[g_{1}\left(t, s, Y^{1}(s), Z^{1}(t, s), K^{1}(t, s, \cdot)\right)\right. \\
\left.-g_{2}\left(t, s, Y^{2}(s), Z^{2}(t, s), K^{2}(t, s, \cdot)\right)\right] d s \\
-\int_{t}^{T} \hat{Z}(t, s) d B(s)-\int_{t}^{T} \int_{\mathbb{R}_{0}} \hat{K}(t, s, \zeta) \tilde{N}(d s, d \zeta), t \in[0, T] .
\end{gathered}
$$

Note that

$$
\begin{aligned}
& g_{1}\left(t, s, Y^{1}(s), Z^{1}(t, s), K^{1}(t, s, \cdot)\right)-g_{2}\left(t, s, Y^{2}(s), Z^{2}(t, s), K^{2}(t, s, \cdot)\right) \\
& =g_{1}\left(t, s, Y^{1}(s), Z^{1}(t, s), K^{1}(t, s, \cdot)\right)-g_{1}\left(t, s, Y^{2}(s), Z^{1}(t, s), K^{1}(t, s, \cdot)\right) \\
& +g_{1}\left(t, s, Y^{2}(s), Z^{1}(t, s), K^{1}(t, s, \cdot)\right)-g_{1}\left(t, s, Y^{2}(s), Z^{2}(t, s), K^{1}(t, s, \cdot)\right) \\
& +g_{1}\left(t, s, Y^{2}(s), Z^{2}(t, s), K^{1}(t, s, \cdot)\right)-g_{1}\left(t, s, Y^{2}(s), Z^{2}(t, s), K^{2}(t, s, \cdot)\right) \\
& g_{1}\left(t, s, Y^{2}(s), Z^{2}(t, s), K^{2}(t, s, \cdot)\right)-g_{2}\left(t, s, Y^{2}(s), Z^{2}(t, s), K^{2}(t, s, \cdot)\right) \\
& \geq \alpha(t, s) \hat{Y}(s)+\beta(s) \hat{Z}(t, s)+\int_{\mathbb{R}_{0}} \theta(s, \zeta) \hat{K}(t, s, \zeta) \nu(d \zeta),
\end{aligned}
$$


where

$$
\alpha(t, s)=\frac{g_{1}\left(t, s, Y^{1}(s), Z^{1}(t, s), K^{1}(t, s, \cdot)\right)-g_{1}\left(t, s, Y^{2}(s), Z^{1}(t, s), K^{1}(t, s, \cdot)\right)}{\hat{Y}(s)} \mathbf{1}_{\{\hat{Y}(s) \neq 0\}},
$$

and

$$
\beta(s)=\frac{g_{1}\left(t, s, Y^{2}(s), Z^{1}(t, s), K^{1}(t, s, \cdot)\right)-g_{1}\left(t, s, Y^{2}(s), Z^{2}(t, s), K^{1}(t, s, \cdot)\right)}{\hat{Z}(t, s)} \mathbf{1}_{\{\hat{Z}(t, s) \neq 0\}} .
$$

Hence

$$
\begin{gathered}
\hat{Y}(t) \geq \hat{\psi}(t)+\int_{t}^{T}\left[\alpha(t, s) \hat{Y}(s)+\beta(s) \hat{Z}(t, s)+\int_{\mathbb{R}_{0}} \theta(s, t, \zeta) \hat{K}(t, s, \zeta) \nu(d \zeta)\right] d s \\
-\int_{t}^{T} \hat{Z}(t, s) d B(s)-\int_{t}^{T} \int_{\mathbb{R}_{0}} \hat{K}(t, s, \zeta) \tilde{N}(d s, d \zeta), t \in[0, T] .
\end{gathered}
$$

We proceed as in Theorem 3.1 and obtain

$$
\hat{Y}(t) \geq \frac{\mathbb{E}\left[\frac{d \mathbb{Q}}{d \mathbb{P}}\left\{\hat{\psi}(t)+\int_{t}^{T} \Phi(t, r) \hat{\psi}(r) d r\right\} \mid \mathcal{F}_{t}\right]}{\mathbb{E}\left[\frac{d \mathbb{Q}}{d \mathbb{P}} \mid \mathcal{F}_{t}\right]},
$$

where $\Phi(t, r)=\sum_{n=1}^{\infty} \alpha^{(n)}(t, r)$.

By hypothesis (3.5), we get that $\alpha(t, r) \geq 0$ for all $t, r$. Hence $\Phi(t, r) \geq 0$ for all $t, r$ together with (3.6) implies that $\hat{Y}(t) \geq 0 \mathbb{P}$-a.s.

The following particular case is essential for the next section.

\subsection{BSVIEs with drivers independent of $Y$}

In this subsection, we are interested in BSVIEs with drivers $g$ independent of $Y$, as follows:

$$
\begin{gathered}
Y(t)=-\psi(t)+\int_{t}^{T} g(t, s, Z(t, s), K(t, s, \cdot)) d s-\int_{t}^{T} Z(t, s) d B(s) \\
-\int_{t}^{T} \int_{\mathbb{R}_{0}} K(t, s, \zeta) \tilde{N}(d s, d \zeta), t \in[0, T] .
\end{gathered}
$$

We impose the following set of assumptions.

- The driver $g: \triangle \times \mathbb{R} \times L_{\nu}^{2} \times \Omega \rightarrow \mathbb{R}$ satisfies

$$
\mathbb{E}\left[\int_{0}^{T}\left(\int_{t}^{T} g(t, s, 0,0) d s\right)^{2} d t\right]<+\infty .
$$

- There exists a constant $C>0$, such that, for all $(t, s) \in \triangle$,

$$
\begin{aligned}
& \left|g(t, s, z, k(\cdot))-g\left(t, s, z^{\prime}, k^{\prime}(\cdot)\right)\right| \\
& \leq C\left(\left|z-z^{\prime}\right|+\left(\int_{\mathbb{R}_{0}}\left|k(\zeta)-k^{\prime}(\zeta)\right|^{2} \nu(d \zeta)\right)^{\frac{1}{2}}\right),
\end{aligned}
$$

for all $z, z^{\prime} \in \mathbb{R}, k(\cdot), k^{\prime}(\cdot) \in L_{\nu}^{2}$.

- The terminal value $\psi(\cdot) \in L_{\mathcal{F}_{T}}^{2}[0, T]$. 
We know by Theorem 2.1 there exists a unique solution $(Y, Z, K) \in L_{y}^{2} \times L_{z}^{2} \times H_{\nu}^{2}$ for the BSVIE (3.8). Consider the following the particular linear BSVIE with jumps

$$
\begin{gathered}
Y(t):=-\psi(t)+\int_{t}^{T}\left[\beta(s) Z(t, s)+\int_{\mathbb{R}_{0}} \theta(s, \zeta) K(t, s, \zeta) \nu(d \zeta)\right] d s \\
-\int_{t}^{T} Z(t, s) d B(s)-\int_{t}^{T} \int_{\mathbb{R}_{0}} K(t, s, \zeta) \tilde{N}(d s, d \zeta), t \in[0, T]
\end{gathered}
$$

where $\psi(\cdot) \in L_{\mathcal{F}_{T}}^{2}[0, T]$ and $\left(\beta(s), \theta(s, \zeta) ; 0 \leq s \leq T, \zeta \in \mathbb{R}_{0}\right.$ are given (deterministic) measurable functions of $s$ and $\zeta$, with values in $\mathbb{R}$. For simplicity we assume that these functions are bounded, and we assume that there exists $\varepsilon>0$ such that $\theta(s, \zeta) \geq-1+\varepsilon$ for all $s, \zeta$.

Since the following results are just particular cases of the previous theorems, we will state them without proofs.

Lemma 3.4 The part of the solution $Y(t)$ of the linear BSVIE with jumps (3.11) can be given on its closed formula, as

$$
Y(t)=\frac{-\mathbb{E}\left[\frac{d \mathbb{Q}}{d \mathbb{P}} \psi(t) \mid \mathcal{F}_{t}\right]}{\mathbb{E}\left[\frac{d \mathbb{Q}}{d \mathbb{P}} \mid \mathcal{F}_{t}\right]}
$$

We can also get the comparison theorem for BSVIE with jumps of type (3.8).

Theorem 3.5 (Comparison Theorem ) For $i=1,2$, let $g_{i}: \triangle \times \mathbb{R} \times L_{\nu}^{2} \times \Omega \rightarrow \mathbb{R}$ and $\psi_{1}(t), \psi_{2}(t) \in L_{\mathcal{F}_{T}}^{2}[0, T]$ and let $\left(Y^{i}, Z^{i}, K^{i}\right)_{i=1,2}$ be the solutions of

$$
\begin{aligned}
Y^{i}(t)=-\psi^{i}(t) & +\int_{t}^{T} g_{i}\left(t, s, Z^{i}(t, s), K^{i}(t, s, \cdot)\right) d s-\int_{t}^{T} Z^{i}(t, s) d B(s) \\
& -\int_{t}^{T} \int_{\mathbb{R}_{0}} K^{i}(t, s, \zeta) \tilde{N}(d s, d \zeta), t \in[0, T] .
\end{aligned}
$$

Assume that the driver $\left(g_{i}\right)_{i=1,2}$ is Lipschitz and satisfies

$$
g_{1}\left(t, s, z^{2}, k^{2}\right) \geq g_{2}\left(t, s, z^{2}, k^{2}\right), \forall t, \mathbb{P} \text {-a.s. }
$$

and that there exists a bounded predictable process $\theta(s, \zeta)$ and $a \Pi(\cdot) \in L_{\nu}^{2}$ such that ds $\otimes$ $d \mathbb{P} \otimes \nu(d \zeta)$-a.s.

$$
\theta(s, \zeta) \geq-1+\varepsilon \text { and }|\theta(s, \zeta)| \leq \Pi(\zeta)
$$

and the following inequality holds

$$
\begin{gathered}
g_{1}\left(t, s, Z^{2}(t, s), K^{1}(t, s, \cdot)\right)-g_{1}\left(t, s, Z^{2}(t, s), K^{2}(t, s, \cdot)\right) \\
\geq \int_{\mathbb{R}_{0}} \theta(s, \zeta)\left(K^{1}(t, s, \zeta)-K^{2}(t, s, \zeta)\right) \nu(d \zeta) .
\end{gathered}
$$

Moreover,

$$
\psi_{1}(t) \leq \psi_{2}(t) \text { for each } t \in[0, T], \mathbb{P} \text {-a.s. }
$$

Then $Y^{1}(t) \geq Y^{2}(t) \mathbb{P}$-a.s. for each $t$. 


\section{Dynamic risk measure by means of BSVIE}

As we have seen in the introduction, a natural way to construct a dynamic risk measures by means of a BSVIEs with jumps, is as follows: Define

$$
\rho(t ; \psi(\cdot)):=Y^{-\psi(\cdot)}(t), \quad \text { for all } t \in[0, T],
$$

where $Y$ is the first component of the solution $(Y(t), Z(t, s), K(t, s, \zeta))$ of the BSVIE

$$
\begin{gathered}
Y(t)=-\psi(t)+\int_{t}^{T} g(t, s, Z(t, s), K(t, s, \cdot)) d s-\int_{t}^{T} Z(t, s) d B(s) \\
-\int_{t}^{T} \int_{\mathbb{R}_{0}} K(t, s, \zeta) \tilde{N}(d s, d \zeta), t \in[0, T]
\end{gathered}
$$

where the terminal condition $\psi \in L_{\mathcal{F}_{T}}^{2}(0, T)$ and the generator $g: \triangle \times \mathbb{R} \times L_{\nu}^{2} \times \Omega \rightarrow \mathbb{R}$ satisfies the assumptions (3.9), (3.10), (3.3).

Theorem 4.1 If $\rho$ and $g$ are defined as above then $\rho$ is a convex dynamic risk measure, i.e. the following holds:

(i) Convexity: Suppose that $(z, k(\cdot)) \mapsto g(t, s, z, k(\cdot))$ is convex, i.e.,

$$
\begin{aligned}
& g\left(t, s, \lambda z_{1}+(1-\lambda) z_{2}, \lambda k_{1}(\cdot)+(1-\lambda) k_{2}(\cdot)\right) \\
& \leq g\left(t, s, \lambda z_{1}, \lambda k_{1}(\cdot)\right)+g\left(t, s,(1-\lambda) z_{2},(1-\lambda) k_{2}(\cdot)\right),
\end{aligned}
$$

for all $(t, s) \in \triangle, z_{1}, z_{2} \in \mathbb{R}, k_{1}(\cdot), k_{2}(\cdot) \in \mathbb{R}_{0}$ and $\lambda \in[0,1]$.

Then $\psi(\cdot) \mapsto \rho(t ; \psi(\cdot))$ is convex, i.e.,

$$
\rho\left(t ; \lambda \psi_{1}(\cdot)+(1-\lambda) \psi_{2}(\cdot)\right) \leq \lambda \rho\left(t ; \psi_{1}(\cdot)\right)+(1-\lambda) \rho\left(t ; \psi_{2}(\cdot)\right), t \in[0, T] .
$$

(ii) Monotonicity: If $\psi_{1}(\cdot) \leq \psi_{2}(\cdot)$, then $\rho\left(t ; \psi_{2}(\cdot)\right) \leq \rho\left(t ; \psi_{1}(\cdot)\right)$.

(iii) Translation invariance: If $\psi(\cdot) \in L_{\mathcal{F}_{T}}^{2}(0, T)$ and a constant $a \in \mathbb{R}$. Then $\rho(t ; \psi(\cdot)+$ $a)=\rho(t ; \psi(\cdot))-a$, for each $t \in[0, T]$.

(iv) Past independence: If $\psi(\cdot), \psi^{\prime}(\cdot) \in L_{\mathcal{F}_{T}}^{2}(0, T)$ and $\psi(s)=\psi^{\prime}(s)$ for all $s \in[t, T]$ then $\rho(t ; \psi(\cdot))=\rho\left(t, \psi^{\prime}(\cdot)\right)$.

Proof (i) Convexity: Fix $\lambda \in(0,1)$ and for all $\psi_{1}(\cdot), \psi_{2}(\cdot) \in L_{\mathcal{F}_{T}}^{2}(0, T)$. We want to prove that

$$
\rho\left(t ; \lambda \psi_{1}(\cdot)+(1-\lambda) \psi_{2}(\cdot)\right) \leq \lambda \rho\left(t ; \psi_{1}(\cdot)\right)+(1-\lambda) \rho\left(t ; \psi_{2}(\cdot)\right)
$$

i.e.,

$$
Y^{-\left(\lambda \psi_{1}(\cdot)+(1-\lambda) \psi_{2}(\cdot)\right)}(t) \leq \lambda\left(Y^{-\psi_{1}(\cdot)}(t)\right)+(1-\lambda)\left(Y^{-\psi_{2}(\cdot)}(t)\right)
$$


Set $(\hat{Y}, \hat{Z}, \hat{K}) \in L_{y}^{2} \times L_{z}^{2} \times H_{\nu}^{2}$ solution of the following BSVIE with jumps

$$
\begin{array}{r}
\hat{Y}(t)=-\lambda \psi_{1}(t)-(1-\lambda) \psi_{2}(t)+\int_{t}^{T} g(t, s, \hat{Z}(t, s), \hat{K}(t, s, \cdot)) d s \\
-\int_{t}^{T} \hat{Z}(t, s) d B(s)-\int_{t}^{T} \int_{\mathbb{R}_{0}} \hat{K}(t, s, \zeta) \tilde{N}(d s, d \zeta), t \in[0, T] .
\end{array}
$$

Define

$$
\begin{array}{ll}
\tilde{Y}(t) & :=\lambda Y^{-\psi_{1}(\cdot)}(t)+(1-\lambda) Y^{-\psi_{2}(\cdot)}(t) \\
\tilde{Z}(t, s) & :=\lambda Z^{-\psi_{1}(\cdot)}(t, s)+(1-\lambda) Z^{-\psi_{2}(\cdot)}(t, s) \\
\tilde{K}(t, s, \cdot) & :=\lambda K^{-\psi_{1}(\cdot)}(t, s, \cdot)+(1-\lambda) K^{-\psi_{2}(\cdot)}(t, s, \cdot) .
\end{array}
$$

Then

$$
\begin{aligned}
& \tilde{Y}(t)=-\lambda \psi_{1}(t)-(1-\lambda) \psi_{2}(t) \\
& +\int_{t}^{T}\left[\lambda g\left(t, s, Z^{-\psi_{1}(\cdot)}(t, s), K^{-\psi_{1}(\cdot)}(t, s, \cdot)\right)+(1-\lambda) g\left(t, s, Z^{-\psi_{2}(\cdot)}(t, s), K^{-\psi_{2}(\cdot)}(t, s, \cdot)\right)\right] d s \\
& \quad-\int_{t}^{T} \tilde{Z}(t, s) d B(s)-\int_{t}^{T} \int_{\mathbb{R}_{0}} \tilde{K}(t, s, \zeta) \tilde{N}(d s, d \zeta) \\
& \geq-\lambda \psi_{1}(t)-(1-\lambda) \psi_{2}(t)+\int_{t}^{T} g(t, s, \tilde{Z}(t, s), \tilde{K}(t, s, \cdot)) \\
& \quad-\int_{t}^{T} \tilde{Z}(t, s) d B(s)-\int_{t}^{T} \int_{\mathbb{R}_{0}} \tilde{K}(t, s, \zeta) \tilde{N}(d s, d \zeta), t \in[0, T]
\end{aligned}
$$

where we have used the convexity of $g$ in the last inequality. By the comparison Theorem 3.5 , we deduce that

$$
\hat{Y}(t) \leq \tilde{Y}(t) \text {, for each } t \in[0, T]
$$

Hence

$$
\begin{aligned}
\rho\left(t ; \lambda \psi_{1}(\cdot)+(1-\lambda) \psi_{2}(\cdot)\right) & =\hat{Y}(t) \leq \tilde{Y}(t) \\
& =\lambda Y^{-\psi_{1}(\cdot)}(t)+(1-\lambda) Y^{-\psi_{2}(\cdot)}(t) \\
& =\lambda \rho\left(t ; \psi_{1}(\cdot)\right)+(1-\lambda) \rho\left(t ; \psi_{2}(\cdot)\right) .
\end{aligned}
$$

(ii) Monotonicity: If $\psi_{1}(\cdot) \leq \psi_{2}(\cdot)$, then, by the comparison Theorem $3.5, Y^{-\psi_{2}(\cdot)}(t) \leq$ $Y^{-\psi_{1}(\cdot)}(t)$. Consequently,

$$
\rho\left(t ; \psi_{2}(\cdot)\right)=Y^{-\psi_{2}(\cdot)}(t) \leq Y^{-\psi_{1}(\cdot)}(t)=\rho\left(t ; \psi_{1}(\cdot)\right) .
$$

(iii) Translation invariant: If $\psi(\cdot) \in L_{\mathcal{F}_{T}}^{2}(0, T)$ and $a \in \mathbb{R}$ is a real constant. Then we get that

$$
Y^{-\psi(\cdot)+a}(t)=Y^{-\psi(\cdot)}(t)+a .
$$

Thus,

$$
\begin{aligned}
\rho(t ; \psi(\cdot)+a) & =Y^{-\psi(\cdot)+a}(t)=Y^{-\psi(\cdot)}(t)-a \\
& =\rho(t ; \psi(\cdot))-a, \text { for each } t \in[0, T] .
\end{aligned}
$$

(iv) The past independence is a direct consequence of the definition of $\rho$. 


\section{Semimartingale issues}

In this section, we will discuss some particular cases where the solution $Y$ of the above BSVIE can be a semimartingale.

For simplicity, we do not consider jumps, since the jump terms do not play an essential role here.

Consider the solution couple $(Y, Z) \in L_{y}^{2} \times L_{z}^{2}$ of a BSVIE of the form

$$
Y(t)=\psi(t)+\int_{t}^{T} g(t, s, Y(s), Z(t, s)) d s-\int_{t}^{T} Z(t, s) d B(s), 0 \leq t \leq T,
$$

where $g: \triangle \times \mathbb{R} \times \mathbb{R} \times \Omega \rightarrow \mathbb{R}$ is a Lipschitz driver and the terminal value $\psi(t) \in L_{\mathcal{F}_{T}}^{2}[0, T]$. In what follows, we denote by the semimartingale $X(t)$ the solution of the stochastic differential equation

$$
X(t)=x_{0}+\int_{0}^{t} b(s, X(s)) d s+\int_{0}^{t} \sigma(s, X(s)) d B(s), t \in[0, T] .
$$

\section{Type 1 - BSVIE}

Let the couple $(Y, Z) \in L_{y}^{2} \times L_{z}^{2}$ be solution of the following BSVIE

$$
Y(t)=F(X(t), X(T))-\int_{t}^{T} Z(t, s) d B(s),
$$

for some function $F: \mathbb{R}^{2} \rightarrow \mathbb{R}$ and $X(t)$ as given above by (5.2).

Now define

$$
F(X(t), X(T)):=F_{1}(X(t)) F_{2}(X(T)),
$$

for functions $F_{1}: \mathbb{R} \rightarrow \mathbb{R}$ and $F_{2}: \mathbb{R} \rightarrow \mathbb{R}$ which are assumed to be twice continuously differentiable $\left(C^{2}\right)$. Consider

$$
Y(t):=F_{1}(X(t)) \tilde{Y}(t)
$$

where $\tilde{Y}(t)$ is the solution of the BSDE

$$
\tilde{Y}(t):=F_{2}(X(T))-\int_{t}^{T} Z(s) d B(s),
$$

and

$$
Z(t, s):=F_{1}(X(t)) Z(s) .
$$

By the Itô formula, we get that $Y(t)$ solution of (5.3) is a semimartingale.

\section{Type 2 - BSVIE}

Similarly as in the previous case, we consider a BSVIE of the form

$$
Y(t)=F(X(t), X(T))-\int_{t}^{T} Z(t, s) d B(s),
$$


for functions $F \in C^{2}\left(\mathbb{R}^{2}\right)$. Then, for

$$
\tilde{Y}(t, x)=F(x, X(T))-\int_{t}^{T} \tilde{Z}(s, x) d B(s),
$$

we have that

$$
Y(t):=\tilde{Y}(t, X(t)), Z(t, s):=\tilde{Z}(s, X(t)) .
$$

Using the Itô-Ventzell formula, we obtain that $Y(t)$ given by (5.4) is a semimartingale.

\section{Type 3 - BSVIE}

Now we consider a BSVIE for a driver $g$ which does not depend on $Z$, as follows:

$$
Y(t)=F(X(t), X(T))+\int_{t}^{T} g(X(t), X(s), Y(s)) d s-\int_{t}^{T} Z(t, s) d B(s), t \in[0, T] .
$$

Knowing $Y$, we can consider

$$
\tilde{Y}(t, x)=F(x, X(T))+\int_{t}^{T} g(x, X(s), Y(s)) d s-\int_{t}^{T} \tilde{Z}(s, x) d B(s), t \in[0, T] .
$$

Define

$$
\begin{aligned}
\bar{Y}(t) & :=\tilde{Y}(t, X(t)), \\
\bar{Z}(t, s) & :=\tilde{Z}(s, X(t)) .
\end{aligned}
$$

Then

$$
\bar{Y}(t)=F(X(t), X(T))+\int_{t}^{T} g(X(t), X(s), Y(s)) d s-\int_{t}^{T} \bar{Z}(t, s) d B(s), t \in[0, T] .
$$

By uniqueness of the solution, we have

$$
\begin{gathered}
Y(t)=\bar{Y}(t)=\tilde{Y}(t, X(t)), \\
Z(t, s)=\bar{Z}(t, s)=\tilde{Z}(s, X(t)) .
\end{gathered}
$$

By Itô-Ventzel's formula, we get that $Y(t)=\tilde{Y}(t, X(t))$ is a semimartingale.

The most general case, i.e., when the driver depends on both $Y$ and $Z$ is still open and it is a subject of further research.

Acknowledgement. We would like to thank Prof. Yong for pointing out the reference [21] which helped us to improve the paper. We also want to thank Prof. Rosazza Gianin for helpful comments. 


\section{References}

[1] Agram, N., \& Øksendal, B. (2015). Malliavin calculus and optimal control of stochastic Volterra equations. Journal of Optimization Theory and Applications, 167(3), 10701094.

[2] Agram, N., Øksendal, B., \& Yakhlef, S. (2018). Optimal control of forward-backward stochastic Volterra equations. In F. Gesztezy et al (editors): Partial Differential equations, Mathematical Physics, and Stochastic Analysis. A Volume in Honor of Helge Holden's 60th Birthday. EMS Congress Reports.

[3] Agram, N., Øksendal, B., \& Yakhlef, S. (2018). New approach to optimal control of stochastic Volterra integral equations. Stochastics An International Journal of Probability and Stochastic Processes. DOI: 10.1080/17442508.2018.1557186

[4] Barrieu, P., \& El Karoui, N. (2004). Optimal derivatives design under dynamic risk measures. Contemporary Mathematics, 351, 13-26.

[5] Bismut, J. M. (1978). An introductory approach to duality in optimal stochastic control. SIAM review, 20(1), 62-78.

[6] Duffie, D., \& Epstein, L. G. (1992). Stochastic differential utility. Econometrica: Journal of the Econometric Society, 353-394.

[7] El Karoui, N., Peng, S., \& Quenez, M. C. (1997). Backward stochastic differential equations in finance. Mathematical finance, 7(1), 1-71.

[8] Föllmer, H., \& Schied, A. (2011). Stochastic finance: an introduction in discrete time. Walter de Gruyter.

[9] Frittelli, M., \& Rosazza Gianin, E. (2004). Dynamic convex risk measures. In: Szego, G. (Ed.), Risk Measures for the 21st Century. J. Wiley, pp. 227-248.

[10] Hu, Y., \& Øksendal, B. (2019). Linear backward stochastic Volterra equations. Stochastic Processes and their Applications, 129(2), 626-633.

[11] Kromer, E., \& Overbeck, L. (2017). Differentiability of BSVIEs and dynamic capital allocations. International Journal of Theoretical and Applied Finance, 20(07), 1750047.

[12] Øksendal, B., \& Sulem, A. (2015). Risk minimization in financial markets modeled by Itô-Lévy processes. Afrika Matematika, 26(5-6), 939-979.

[13] Pardoux, E., \& Peng, S. (1992). Backward stochastic differential equations and quasilinear parabolic partial differential equations. In Stochastic partial differential equations and their applications (pp. 200-217). Springer. 
[14] Peng, S. (2004). Nonlinear expectations, nonlinear evaluations and risk measures. In Stochastic methods in finance (pp. 165-253). Springer.

[15] Quenez, M. C., \& Sulem, A. (2013). BSDEs with jumps, optimization and applications to dynamic risk measures. Stochastic Processes and their Applications, 123(8), 33283357 .

[16] Quenez, M. C., \& Sulem, A. (2014). Reflected BSDEs and robust optimal stopping for dynamic risk measures with jumps. Stochastic Processes and their Applications, 124(9), 3031-3054.

[17] Ren, Y. (2010). On solutions of backward stochastic Volterra integral equations with jumps in Hilbert spaces. Journal of optimization theory and applications, 144(2), 319333.

[18] Rosazza Gianin, E. (2006). Risk measures via g-expectations. Insurance: Mathematics and Economics, 39(1), 19-34.

[19] Royer, M. (2006). Backward stochastic differential equations with jumps and related non-linear expectations. Stochastic Processes and their Applications, 116(10), 13581376 .

[20] Tang, S., \& Li, X. (1994). Necessary conditions for optimal control of stochastic systems with random jumps. SIAM Journal on Control and Optimization, 32(5), 1447-1475.

[21] Wang, T., \& Yong, J. (2015). Comparison theorems for some backward stochastic Volterra integral equations. Stochastic Processes and their Applications, 125(5), 17561798.

[22] Wang, Z., \& Zhang, X. (2007). Non-Lipschitz backward stochastic Volterra type equations with jumps. Stochastics and Dynamics, 7(04), 479-496.

[23] Yong, J. (2006). Backward stochastic Volterra integral equations and some related problems. Stochastic Processes and their Applications, 116(5), 779-795.

[24] Yong, J. (2008). Well-posedness and regularity of backward stochastic Volterra integral equations. Probability Theory and Related Fields, 142(1-2), 21-77.

[25] Yong, J. (2013). Backward stochastic Volterra integral equations - a brief survey. Applied Mathematics-A Journal of Chinese Universities, 28(4), 383-394.

[26] Yong, J. (2007). Continuous-time dynamic risk measures by backward stochastic Volterra integral equations. Applicable Analysis, 86(11), 1429-1442. 